\title{
Robust Fractional Model Predictive Controller (FMPC) Design for Under-Actuated Robotic Systems
}

\author{
Abhaya Pal Singh ${ }^{1, *}$, Himanshu Agrawal ${ }^{2}$ and Pallavi Srivastava ${ }^{3}$ \\ 1,*Research Scholar, Symbiosis Institute of Technology, Symbiosis International \\ University, Pune, India \\ ${ }^{2}$ Symbiosis Institute of Technology, Symbiosis International University, Pune, \\ India \\ ${ }^{3}$ Department of Mechatronics, Symbiosis Skills and Open University, Pune, India \\ *abhaya.aps@gmail.com
}

\begin{abstract}
In this paper a novel robust fractional model predictive controller is designed for under-actuated robotic system. The considered under-actuated robotic system is an inverted pendulum on a cart system with two degree of freedom and a control input. The system is modeled to its fractional equivalent model and approximated from the Oustaloup-Recursive-Approximation. From the approximated model a best model is chosen and fractional model predictive controller is designed. Robustness of the fractional model predictive controller is checked to the variations of the system parameters of inverted pendulum on a cart system.
\end{abstract}

Keywords: Fractional controller, Fractional model predictive controller, Robustness, Robotic systems, Under-actuated systems, Model predictive control

\section{Introduction}

In various real world applications upright balance is needed. For example, humanoid and biped robots, two wheeled autonomous robots, rocket or missile guidance etc. The control of these systems is very challenging because it is required to balance them to maintain their vertical position without fail. The best example one can think of is an inverted pendulum on a cart (POAC) which is the foundation of all the robots in under-actuated upright balance category. The POAC is a benchmark problem in the field of control and dynamical theory and this widely used in testing various control related algorithms such as- PID controllers [1, 25] neural networks [3], fuzzy control [2], genetic algorithms [4], PSO, PID based [18], etc. In this paper this benchmark problem is considered and the author tried to give a novel fractional model predictive control (FMPC) for the POAC. The purpose of this FMPC controller is to provide best control of the considered system as well as robustness of the considered system.

Prediction of control is the collection of various techniques to control which optimizes certain properties by the help of model which predict systems behavior to determine sequence of control for further events. Model Predictive Control (MPC) takes a lot variety of models to provide flexibility in handling industrial processes [10], [13]. Many applications of Model Predictive Control (MPC) in the area related to under-actuated systems have been studied in past, e.g., Performance as well as computation efficiency of MPC controlled crane is demonstrated with experimental and valid simulation in [11], adaptive control strategy for control of flexible crane is reported in [12]. It is well known

Received (October 6, 2017), Review Result (December 6, 2017), Accepted (December 12, 2017)

* Corresponding Author 
with the literature of fractional calculus that the fractional control is better control as compared to the traditional control of under-actuated systems [5, 6]. MPC of fractional order systems is reported in [7]. An adaptive MPC based on fractional model is reported in [8] and it was observed that the dynamic response is very smooth and quick. A robust MPC for general systems is developed in [9] for better response depending on fractional model. Hence, using all these merits, this paper proposes a FMPC for controlling the position and angle of POAC system. FMPC performance is compared with classical MPC control to validate that the FMPC controller is better than classical MPC. Furthermore, the robustness of fractional model predictive controller is verified regarding the variation of the parameters of POAC system.

\section{Modeling and Fractional Embedding to the System}

POAC has two degrees of freedom and one control input. This system is unstable if control is not provided. This system has a pendulum mounted on a cart supported with a rod. The cart can move only in one direction which is along $x-$ axis. The POAC system is shown in Figure 1. Let us take $M$ as the mass of cart and $m_{\text {is mass }}$ of the pendulum with the rod length of $l$. Assuming the mass of the rod and friction between the surface and wheel is negligible.

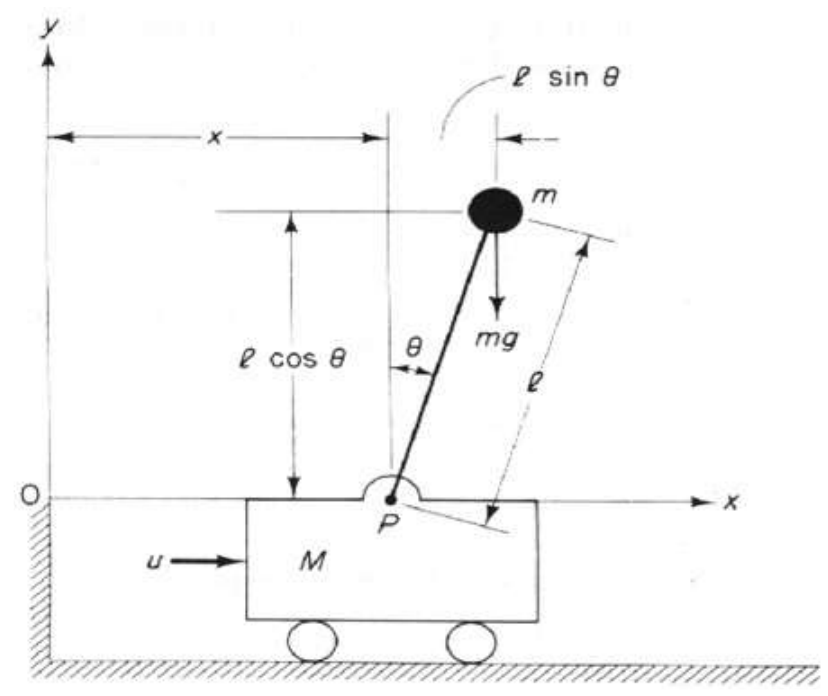

Figure 1. POAC System

Let $g$ be the acceleration due to gravity, ${ }^{x(t)}$ be the position along the axis and $\theta(t)$ be the angle between the vertical axis the rod. The modeling equations can be derived using Euler-Lagrange formulation [14], [24]. The Lagrangian is the difference between total kinetic energy KE of the system to the total potential energy PE of the system.

$$
L=K E-P E
$$

After calculating the values of KE and potential PE of the system the Lagrangian equation will be:-

$$
L=\frac{1}{2}(M+m) \dot{x}^{2}+\frac{1}{2} m\left(l^{2} \dot{\theta}^{2}+2 \dot{x} \dot{\theta} l \cos \theta\right)-m g l \cos \theta
$$


To find equation of motion, equation (2) should be considered and putting this equation to Euler-Lagrange formulation further after some mathematical manipulation [19] and transforming to its equivalent linear state space model, we get:-

$$
\left[\begin{array}{c}
\dot{x} \\
\ddot{x} \\
\dot{\theta} \\
\ddot{\theta}
\end{array}\right]=\left[\begin{array}{cccc}
0 & 1 & 0 & 0 \\
0 & 0 & \frac{-m g}{M} & 0 \\
0 & 0 & 0 & 1 \\
0 & 0 & \frac{(M+m)}{M l} & 0
\end{array}\right]\left[\begin{array}{l}
x \\
\dot{x} \\
\theta \\
\dot{\theta}
\end{array}\right]+\left[\begin{array}{l}
0 \\
\frac{1}{M} \\
0 \\
\frac{-1}{M l}
\end{array}\right][u]
$$

And the output as:-

$$
y=\left[\begin{array}{llll}
1 & 0 & 0 & 0 \\
0 & 0 & 1 & 0
\end{array}\right]\left[\begin{array}{c}
x \\
\dot{x} \\
\theta \\
\dot{\theta}
\end{array}\right]
$$

Equation (3) and (4) are the linear state space model of POAC system. This system is further considered for the controller design. Let us convert the state space model to its equivalent transfer function model and taking $\mathrm{M}=0.5 \mathrm{~kg}, \mathrm{~m}=0.2 \mathrm{~kg}, 1=1 \mathrm{~m}$ and $\mathrm{g}=9.8 \frac{\mathrm{m}}{\mathrm{s}^{2}}$, the model will be:-

$$
H(s)=\left[\begin{array}{c}
\frac{2 s^{2}-19.6}{s^{4}-13.72 s^{2}} \\
\frac{-2 s^{2}}{s^{4}-13.72 s^{2}}
\end{array}\right]
$$

The fractional equivalent transfer function of equation (5) can be expressed as equation (6) by opting the strategy used in the papers [20], [15] the

$$
H\left(s^{\alpha}\right)=\left[\begin{array}{c}
\frac{2 s^{2 \alpha}-19.6}{s^{4 \alpha}-13.72 s^{2 \alpha}} \\
\frac{-2 s^{2 \alpha}}{s^{4 \alpha}-13.72 s^{2 \alpha}}
\end{array}\right]
$$

The fractional order model is considered because fractional order systems are more flexible when compared to integer order systems. There are many papers/books that validates the concept behind using fractional order models [21], [22] and this is the reason behind considering fractional model as expressed in equation (6) of inverted pendulum on a cart system.

Clearly it can be observed that if we put $\alpha=1$, then equation (5) and equation (6) are same. Using $\alpha$ the fractional term is embedded to the transfer function of POAC system. Depending on the values of $\alpha$, a valid model is derived. Let us take some cases on $\alpha$ for analysis and assuming $0<\alpha \leq 1$.

Case 1. If $\alpha=0.3$, the equivalent transfer function from equation (6) can be written as:- 


$$
H\left(s^{0.3}\right)=\left[\begin{array}{c}
\frac{2 s^{0.6}-19.6}{s^{1.2}-13.72 s^{0.6}} \\
\frac{-2 s^{0.6}}{s^{1.2}-13.72 s^{0.6}}
\end{array}\right]
$$

This equation (7) is fractional equivalent model of POAC system. Approximating this equation using Oustaloup recursive approximation [16], it is:-

$$
\overline{H(s)}=\left[\begin{array}{c}
\frac{s+90}{s^{2}+2 s+1} \\
\frac{s+0.12}{s^{2}+2 s+1}
\end{array}\right]
$$

Equation (8) represents the same model as equation (7) with the difference that equation (8) is approximated model of equation (7), hence if we design the controller corresponding to this model then we are designing a controller corresponding to the fractional model of POAC system with $\alpha=0.3$.

Similarly we can take other values of $\alpha_{\text {to derive the fractional model as in Case }}$ 2. If $\alpha=0.5$, Case 3. If $\alpha=0.2$, Case 4. If $\alpha=0.8$ and Case 5. If $\alpha=1$, which corresponds to the original model of inverted pendulum on a cart system.

\section{Control Strategy of FMPC}

First time Fractional Model Predictive (FMPC) control strategy is implemented on POAC system. For controller design, MATLAB MPC toolbox [17] is used. Figure 2 shows the structure of MPC toolbox.

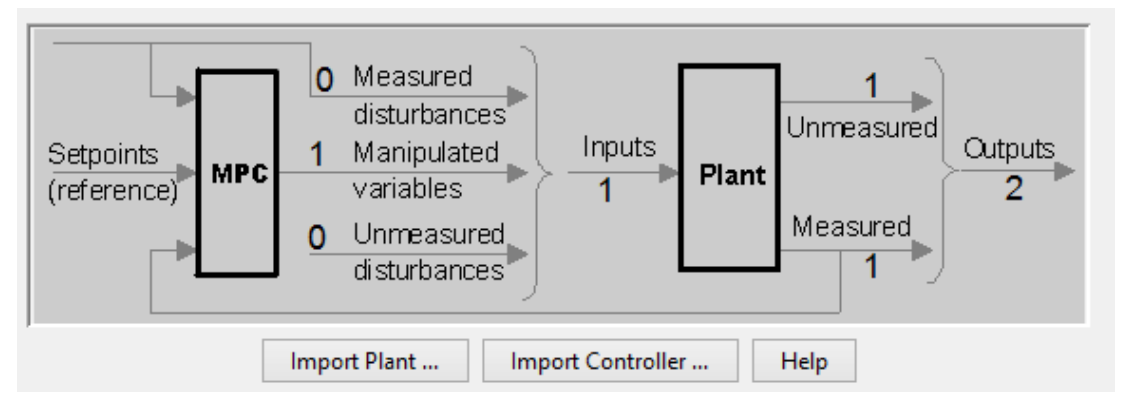

Figure 2. MPC Toolbox Structure

From Figure 2, it can be observed that there is one input which is applied on the cart and two outputs, one is the measured output which is the carts position and the other one is the unmeasured which is the pendulums angle. The aim here is to control the position and angle of pendulum.

Let us take Case 1 to design the fractional model predictive controller using the toolbox shown in figure 2 with sampling time of $0.1 \mathrm{~s}$, prediction horizon to $30 \mathrm{~s}$ and control horizon to $6 \mathrm{~s}$. The aim is to control position $x(t)$ and angle $\theta(t)$ with minimum overshoot and in short interval of time. The response of the controlled system is shown in figure 3 . 


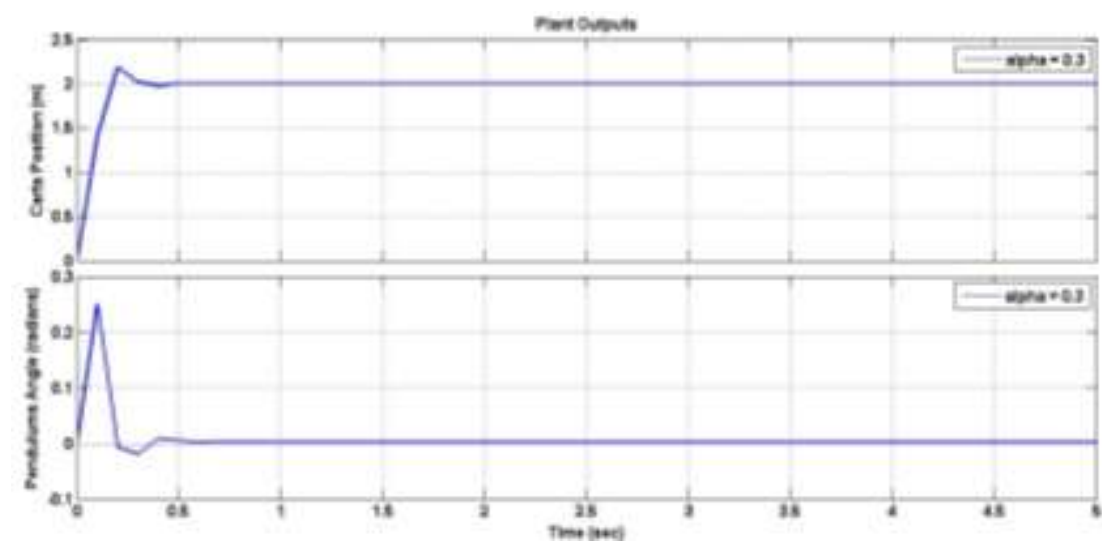

Figure 3.Controlled Output for $\alpha=0.3$

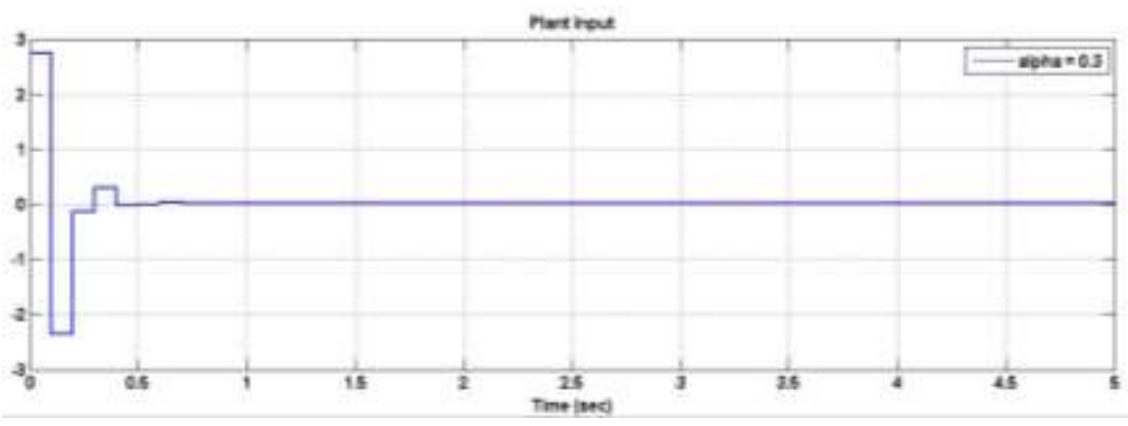

Figure 4. Controlled Input for $\alpha=0.3$

Figure 3 and Figure 4 show the output controlled response and input controlled response of the pendulum on a cart system. It can be observed from the figures that the controller design for $\alpha=0.3$ gives good results. It is having quick settling time in controlling the position and the angle. So the fractional model of POAC corresponding to $\alpha=0.3$ can be used to design fractional model predictive controller which will give stable response.

Let us take Case 5 and using the same condition on the controller as in Case 1 . Case 5 corresponds to the original system i.e., $\alpha=1$. Figure 5 and Figure 6 shows the output and input controlled response of inverted pendulum on a cart system.

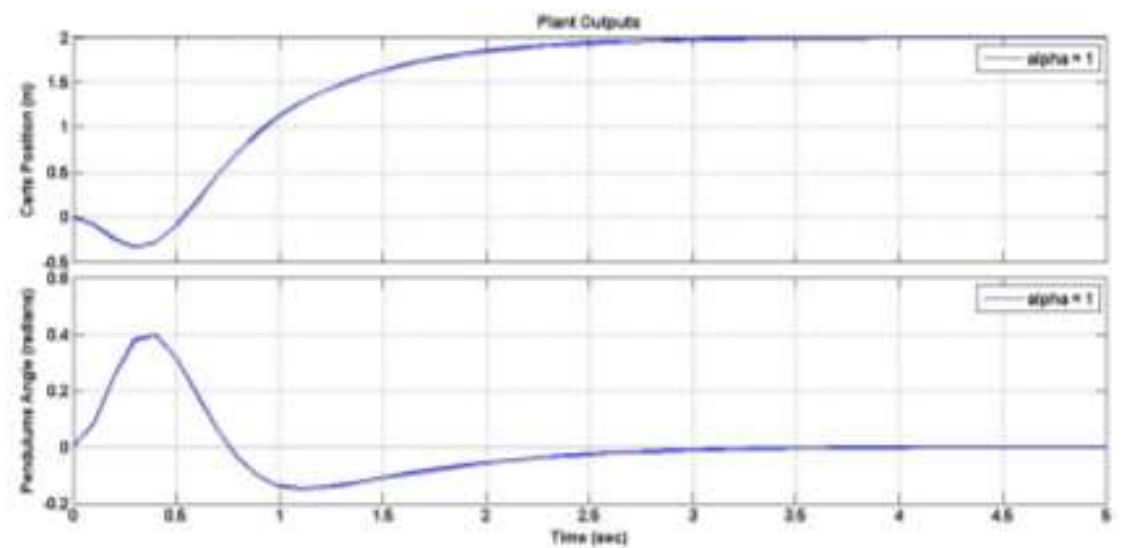

Figure 5.Controlled Output for $\alpha=1$ 


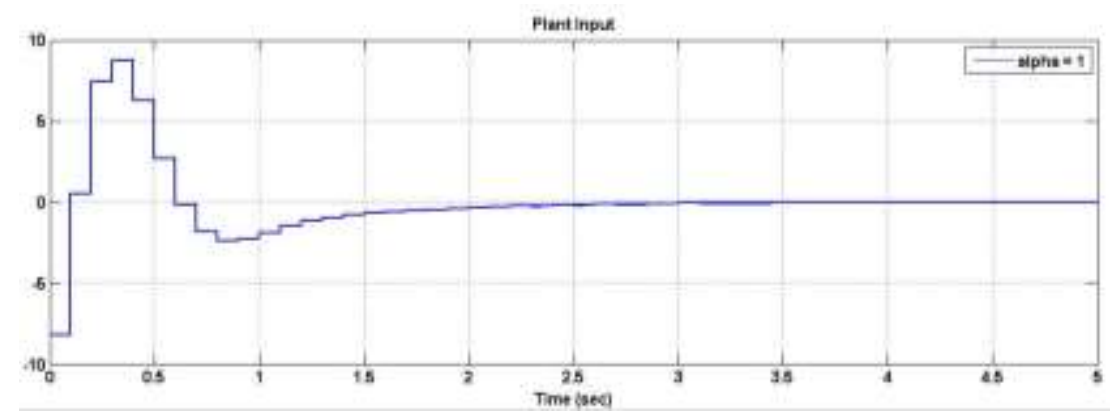

Figure 6. Controlled Input for $\alpha=1$

From Figure 5 and Figure 6 one can conclude that traditional model predictive controller can be used to get controlled response for the considered system.

The dynamic response of FMPC in Figure 3 is quick as compared to traditional MPC in Figure 5. On comparing Figure 3 with Figure 5, it can be clearly seen that fractional model predictive controller (FMPC) is best as compared to traditional model predictive controller (MPC). Now, let us take each and every case which we have considered here and design the controller to understand more about the fractional model predictive controller.

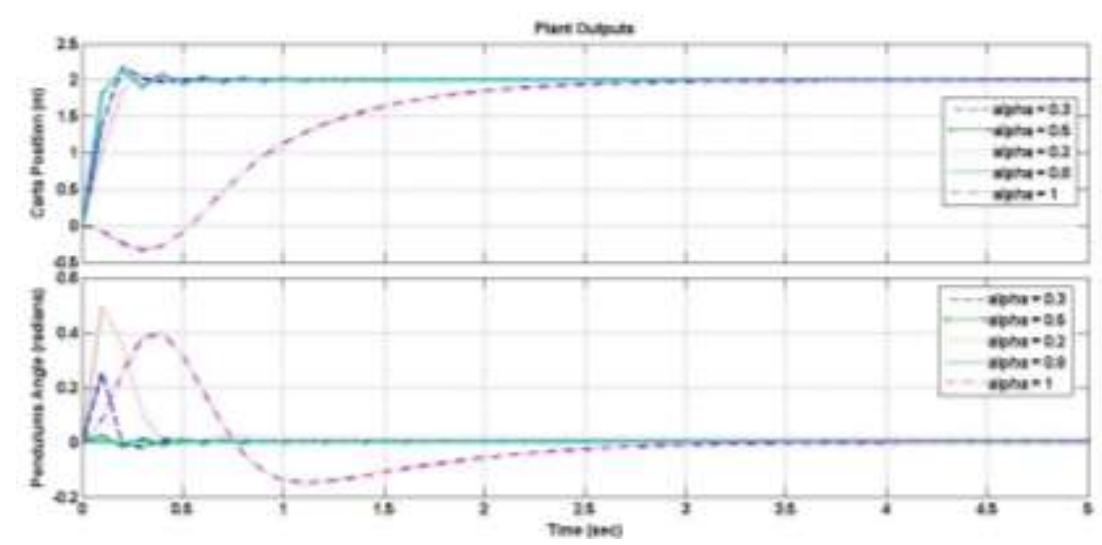

Figure 7. Controlled Output for $\alpha=0.2,0.3,0.5,0.8$ and 1 .

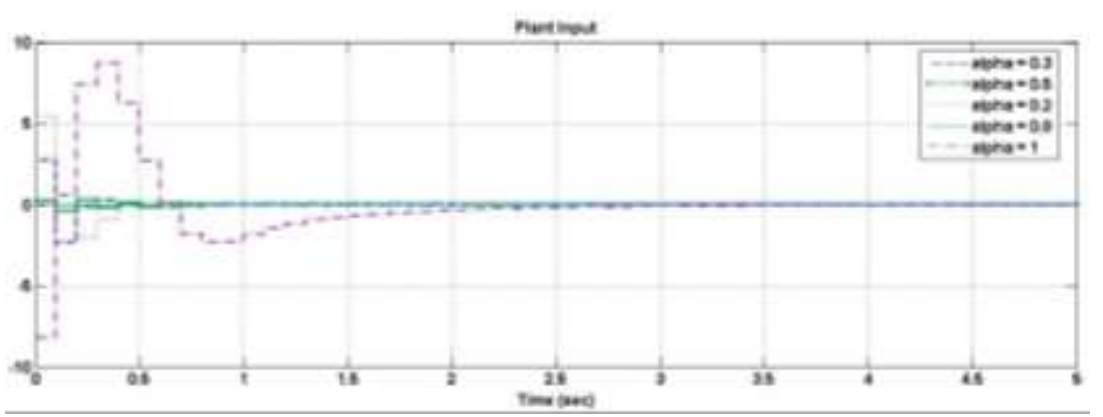

Figure 8. Controlled Input for $\alpha=0.2,0.3,0.5,0.8$ and 1.

Figure 7 and Figure 8 shows the detailed comparison of the FMPC control with traditional MPC control for inverted pendulum on a cart system. Table 1 gives the summery of Figure 7 and Figure 8. 
Table 1. Comparison Table

\begin{tabular}{|c|l|l|l|l|}
\hline $\begin{array}{c}\text { Specific- } \\
\text { ation }\end{array}$ & \multicolumn{2}{|c|}{ Settling Time (sec) } & \multicolumn{2}{l|}{ Oscillations } \\
\hline$\alpha$ & $x(t)$ & $\theta(t)$ & $x(t)$ & $\theta(t)$ \\
\hline 0.2 & 0.49 & 0.84 & no & yes \\
\hline$\underline{\mathbf{0 . 3}}$ & $\underline{\mathbf{0 . 4 2}}$ & $\underline{\mathbf{0 . 6 4}}$ & $\underline{\text { no }}$ & $\underline{\underline{\text { yes }}}$ \\
\hline 0.5 & 1.03 & 1.40 & yes & yes \\
\hline 0.8 & 1.01 & 1.77 & yes & yes \\
\hline$\underline{\mathbf{1}}$ & $\underline{\mathbf{3 . 1 5}}$ & $\underline{\mathbf{3 . 5 5}}$ & $\underline{\text { no }}$ & yes \\
\hline
\end{tabular}

From the comparison table, it can be observed that each fractional model predictive control response is better compared to traditional model predictive controller with $\alpha=1$ in every aspect. If $\alpha=0.3$ then the fractional model predictive controller gives its best result for POAC system. So, $\alpha=1_{\text {in equation }} 6$ gives the optimal model of POAC system to give best response. Let us check the robustness of this fractional model predictive control corresponding to alpha value of 0.3 ( $\alpha=0.3$ ).

\section{Robustness of the Control Strategy to the Change of the System Parameters}

The ability of the system to resist the change without adapting its initial stable configuration is robustness [23]. Let us check the robustness of the Fractional Model Predictive controller for POAC system to the change in the mass of the pendulum and to change in the mass of the cart. Taking two cases one, the change in the mass of cart and the other is the change in the mass of pendulum.

Case 1. When the cart mass is changing.

Let us take the mass of the cart is once $M=30 \mathrm{~kg}$ and other $M=50 \mathrm{~kg}$ and design the fractional model predictive controller for $\alpha=0.3$.

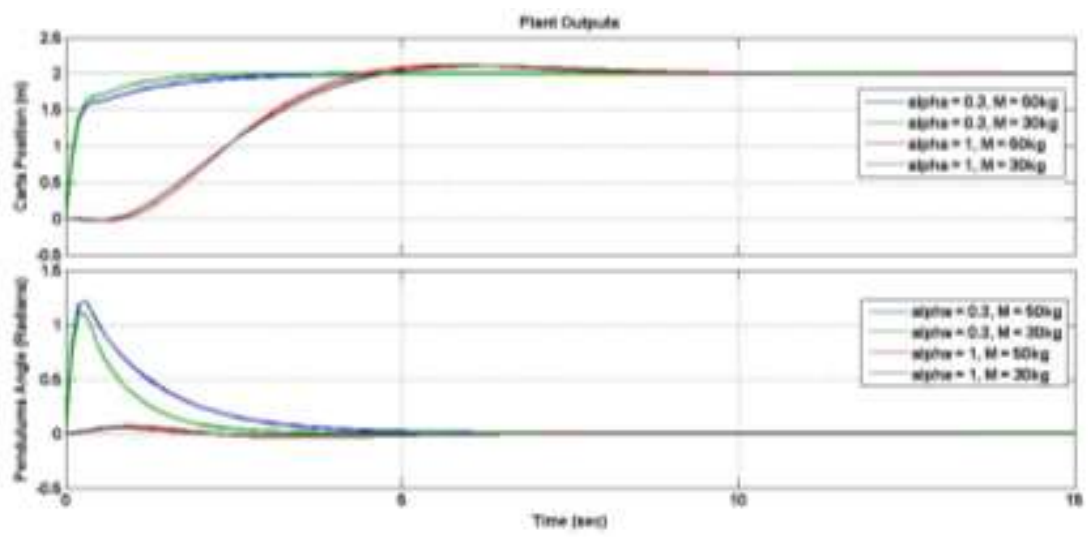

Figure 9. Controlled Output for $\alpha=0.3$ and $\mathrm{M}=50 \mathrm{~kg}, \alpha=0.3$ and $\mathrm{M}=30 \mathrm{~kg}$ 


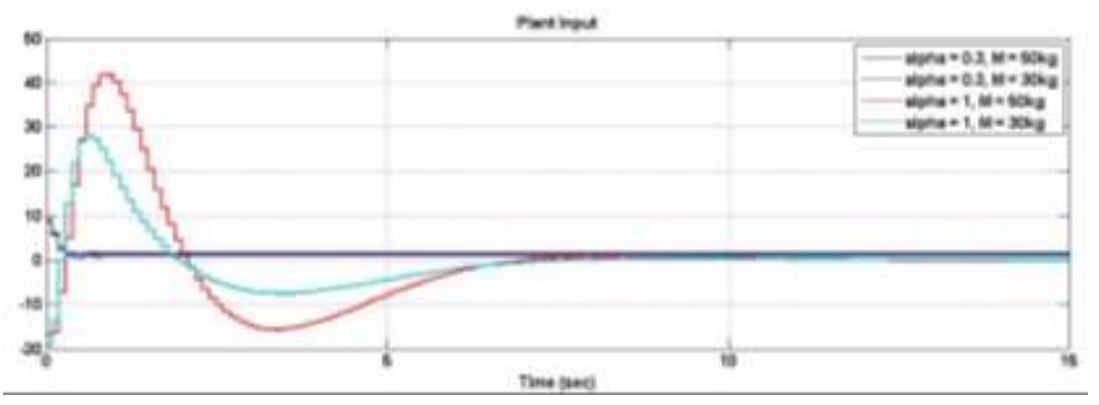

Figure 10. Controlled Input for $\alpha=0.3$ and $M=50 \mathrm{~kg}, \alpha=0.3$ and $M=30 \mathrm{~kg}$

So, from Figure 9 and Figure 10 it is observed that the fractional model predictive controller settles very fast as compared to traditional MPC to change in the mass of the cart and hence FMPC is robust to change in the value of cart mass $M$.

Case 2. When the pendulum mass is changing.

Let us take the mass of the pendulum is once $m=2 \mathrm{~kg}$ and other $m=5 \mathrm{~kg}$ and design the fractional model predictive controller for $\alpha=0.3$.

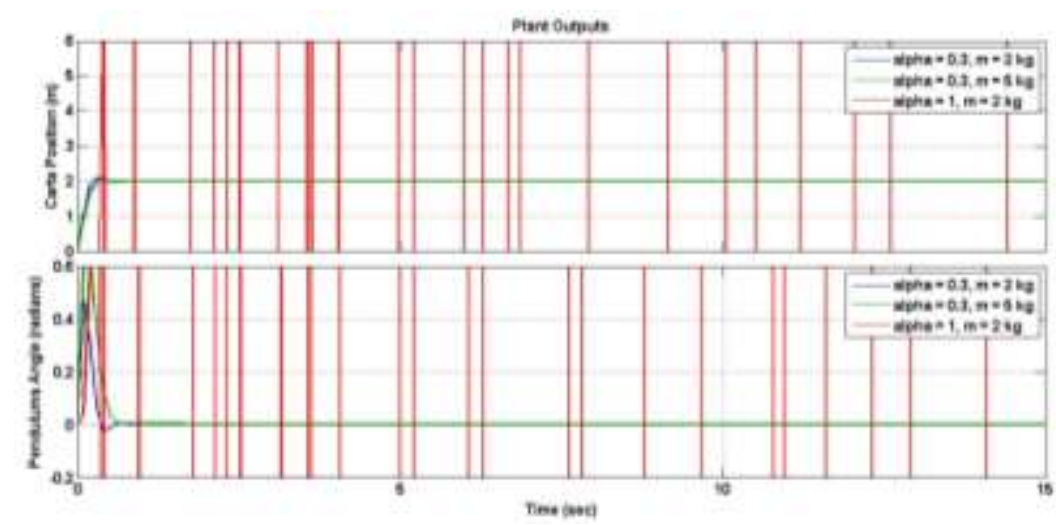

Figure 11. Controlled Output for $\alpha=0.3$ and $\mathrm{m}=2 \mathrm{~kg}, \alpha=0.3$ and $\mathrm{m}=5 \mathrm{~kg}$

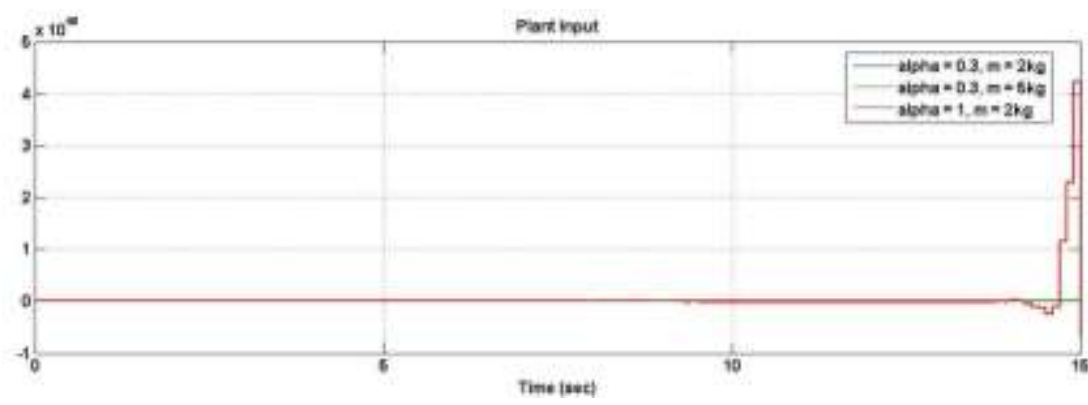

Figure 12. Controlled Input for $\alpha=0.3$ and $\mathrm{m}=2 \mathrm{~kg}, \alpha=0.3$ and $\mathrm{m}=5 \mathrm{~kg}$

So, from Figure 11 and Figure 12 it is observed that the fractional model predictive controller response settles very fast and traditional MPC is unstable to change in the mass of the pendulum and hence it shows that FMPC is robust to change in the value of pendulum mass $m$. 


\section{Conclusion}

The aim of designing a Fractional Model Predictive controller for POAC system is achieved and it is also observed that FMPC control works pretty well for inverted pendulum on a cart system. The proposed FMPC shows better results in all control aspects compared to that traditional MPC. Further, it is also reported in this paper that FMPC is a robust controller to the variations of the system parameters for POAC system and the traditional MPC is unstable to the variation of the mass of the pendulum. Hence this paper reported a novel robust fractional model predictive controller for POAC system.

\section{References}

[1] S. Cong and Y. Liang, "PID-like neural network nonlinear adaptive control for uncertain multivariable motion control systems", Industrial Electronics, IEEE Transactions, vol. 56, no. 10, (2009), pp. 38723879.

[2] J. Yi, N. Yubazaki and K. Hirota, "Upswing and stabilization control of inverted pendulum system based on the SIRMs dynamically connected fuzzy inference model", Fuzzy Sets and Systems, vol. 122, no. 1 , (2001), pp. 139-152.

[3] S. Jung and S. Su Kim, "Control experiment of a wheel-driven mobile inverted pendulum using neural network", Control Systems Technology, IEEE Transactions, vol. 16, no. 2, pp. 297-303.

[4] H. K. Lam, F. H. Frank Leung and P. Kwong-Shun Tam, "Design and stability analysis of fuzzy model based nonlinear controller for nonlinear systems using genetic algorithm", In Fuzzy Systems, FUZZIEEE'02. Proceedings of the 2002 IEEE International Conference on IEEE, vol. 1, (2002), pp. 232-237.

[5] A. P. Singh, S. K. Faruk, M. S. Navdeep and P. Srivastava, "PI $\alpha$ D $\beta$ controller design for underactuated mechanical systems", In Control Automation Robotics \& Vision (ICARCV), 2012 12th International Conference on IEEE, (2012), pp. 1654-1658.

[6] A. P. Singh, H. Agarwal and S. Pallavi, "Fractional Order Controller Design for Inverted Pendulum On A Cart System (POAC)", In WSEAS Transactions on System and Control, vol. 10, (2015), pp. 172-178.

[7] D. Boudjehem and B. Boudjehem, "A fractional model predictive control for fractional order systems", In Fractional dynamics and control, Springer New York, (2012), pp. 59-71.

[8] Z. Deng, H. Cao, X. Li, J. Jiang, J. Yang and Y. Qin, "Generalized predictive control for fractional order dynamic model of solid oxide fuel cell output power", Journal of Power Sources, vol. 195, no. 24, pp. 8097-103, (2010) December 15.

[9] A. Rhouma and F. Bouani, "Robust model predictive control of uncertain fractional systems: a thermal application", IET Control Theory \& Applications, vol. 8, no. 17, (2014), pp. 1986-1994.

[10] J. A. Rossiter, "Model-Based Predictive Control: A Practical Approach", FL: CRC Press, Boca Raton, (2003).

[11] B. Kapernick and K. Graichen, "Model predictive control of an overhead crane using constraint substitution”, In American Control Conference (ACC), IEEE, (2013) June 17, pp. 3973-3978.

[12] W. He, S. Zhang and S. Sam Ge, "Adaptive control of a flexible crane system with the boundary output constraint", Industrial Electronics, IEEE Transactions, vol. 61, no. 8, (2014), pp. 4126-4133.

[13] E. F. Camacho and C. Bord'ons, "Model Predictive Control", Springer-Verlag, Berlin, (2004).

[14] A. P. Singh, F. Kazi, N. M. Singh and V. Vishwesh, "Fractional Order controller design for underactuated mechanical systems", In The 5th IFAC Symposium on Fractional Differentiation and its Applications-FDA, (2012).

[15] T. Srivastava, A. P. Singh and H. Agarwal, "Modeling the Under-Actuated Mechanical System with Fractional Order Derivative", Progress in Fractional Differentiation and Applications, vol. 1, no. 1, (2015), pp. 57-64.

[16] W. Krajewski and U. Viaro, "A method for the integer-order approximation of fractional-order systems", Journal of the Franklin Institute, vol. 351, no. 1, (2014), pp. 555-564.

[17] A. Bemporad, M. Morari and N. Lawrence Ricker, "Model Predictive Control Toolbox 3 User's Guide", The mathworks, (2010).

[18] S. Howimanporn and M. Parnichkun, "Control of an X-Y Planar Inverted Pendulum using PSO-BASED SMC", International Journal of Robotics and Automation, vol. 30, no. 4, (2015).

[19] A. P. Singh and H. Agrawal, "On Modeling and Analysis of Launch Vehicle System", In Proceedings of the International Conference on Signal, Networks, Computing, and Systems, Lecture notes in electrical engineering, Springer India, vol. 396, (2016), pp. 273-279.

[20] A. Dzieliński, G. Sarwas and D. Sierociuk, "Comparison and validation of integer and fractional order ultracapacitor models", Advances in Difference Equations, (2011) December.

[21] W. Khan, "A comparison between integer and fractional order algebraic approaches for on-line parameter estimation and position control of DC motor", Proceedings of 2014 11th International 
Bhurban Conference on Applied Sciences \& Technology (IBCAST) Islamabad, Pakistan, IEEE, (2014) January 14th-18th.

[22] E. Ahmed, A. M. A. El-Sayed and H. AA El-Saka, "Equilibrium points, stability and numerical solutions of fractional-order predator-prey and rabies models", Journal of Mathematical Analysis and Applications, vol. 325, no. 1, (2007), pp. 542-553.

[23] C. Alippi, "Robustness Analysis chapter in Intelligence for Embedded Systems", Springer, ISBN 978-3319-05278-6, (2014), pp. 283.

[24] A. P. Singh, T. Srivastava, H. Agrawal and P. Srivastava, "Fractional Order Controller Design and Analysis for Crane System”, Progress in Progr. Fract. Differ. Appl., vol. 3, no. 2, (2017) April, pp. 155162.

[25] S. Joshi and A. P. Singh, "Comparison of the performance of controllers for under-actuated systems", 2016 IEEE 1st International Conference on Power Electronics, Intelligent Control and Energy Systems (ICPEICES), Delhi, (2016), pp. 1-5. 